\title{
ROBUSTNESS OF OPTIMAL DESIGN SOLUTIONS TO REDUCE VIBRATION TRANSMISSION IN A LIGHTWEIGHT 2-D STRUCTURE, PART I: GEOMETRIC DESIGN
}

\author{
D. K. Anthony and S. J. Elliott \\ Institute of Sound and Vibration Research, University of Southampton, Highfield, \\ Southampton SO17 1BJ, England \\ AND \\ A. J. KEANE \\ Department of Mechanical Engineering, University of Southampton, Highfield, \\ Southampton SO17 1BJ, England
}

(Received 8 October 1998, and in final form 14 July 1999)

A two-dimensional lightweight cantilever structure is studied, comprising 40 rigidly joined beams, of which the geometry is optimized to reduce vibration transmission over a given bandwidth. In this paper, the optimization is achieved by using genetic algorithms. Ten optimized design candidates were achieved for each of three cases resulting from minimizing an objective function (the vibration transmission between two points on the structure) which is calculated (i) using a single frequency, (ii) the frequency average over a narrow bandwidth, and (iii) the frequency average over a broad frequency range. All the candidates show performance improvements and normally the best performance is taken to be the best candidate. This paper then considers the sensitivity of each optimal candidate to small changes in the geometry of the structure. If the performance of a structure is too sensitive to perturbations its practical application is limited or may not be realizable in practice. The robustness of the optimized candidates is studied in order to find those candidates which are least sensitive to changing design parameters. This is achieved by perturbing the positions of the joints by an ensemble of sets of random numbers. The statistical effect on the objective function is investigated, and some candidates are seen to be more robust to such perturbations than others and generally the greater the bandwidth over which the structure is optimized the more robust the design. A selection criterion is then applied which enables the best candidates to be identified on grounds of both nominal optimized performance and robustness. Finally, the advantage of using genetic algorithms over traditional "hill-climbing" optimization methods is shown, on the grounds of both nominal performance and robustness. 


\section{INTRODUCTION}

Unwanted vibration in a structure can have many undesirable effects. It can cause damage to the structure itself or components to which it is connected. It may prevent the structure being used for its intended application if it occurs in critical regions. The example motivating the study here is that of the vibration of an antenna mounted on the end of statellite boom arms. Some structures (especially those employed in space) often have inherently small amounts of damping and the transmission of vibration through the structure can be an important issue. The need is even greater in lightweight structures as the controlling inertial effects of the mass are reduced.

Traditional techniques to reduce vibration are to increase the mass and/or damping of the structure. The former is normally in violation with design goals, and the latter is the most regularly applied passive technique. A more recent development has been the application of active control of vibration systems [1] which act to produce countervibrations in order to reduce vibration. The use of such techniques is investigated in the second companion paper [2]. Alternatively, where applicable and practical, vibration transmission can be reduced by dynamically isolating the structure, again using the aforementioned techniques. It is desirable to consider the dynamic behaviour of the structure during its design. It may be possible to optimize the inherent performance alleviating the need for any additional vibration control measures.

Previous work [3] has considered the optimization of the geometry of a lightweight two-dimensional structure comprised of 40 rigidly joined beams to minimize vibration transmission. The positions of the joints were used as the optimization variables. The design was optimized in order to produce a structure that inherently had a much greater degree of vibration isolation than the original, traditional, regular design (achieving an improvement of three orders of magnitude). The optimization by exhaustive search is computationally expensive for this highly non-linear problem but the use of genetic algorithms was found to provide a reasonably efficient method of finding optimized design candidates. The optimized design was also verified in practice [4]. The optimization was later applied to a three-dimensional structure [5].

Although the optimized designs show better performance in theory, practical design implementations with exactly the required parameters may not be feasible, due to manufacturing tolerances, for example. Even if this was not the case, the parameters might change during service due to, for example, thermal expansion and contraction. If the effect of these changes on the optimized performance is not studied, then a design candidate that is predicted to be the best (under nominal operating conditions) in service may yield less than optimum performance whereas another candidate, although having a slightly lower optimized performance under nominal operating conditions may be less sensitive (more robust) to changing operating conditions, and thus would be a more practical choice.

The work reported here uses the same approach for genetic algorithms to optimize the same structure studied by Keane [3]. Then, the robustness of each of these designs is analyzed, so that designs that are statistically less likely to be 
sensitive to small changes in operating conditions are found. A measure is suggested that may be used in order to rank the likely performance of optimized design candidates taking into account both their nominal performance and robustness.

This paper is structured is follows: section 2 describes the structure which is the subject of this study, section 3 discusses design optimization using evolutionary algorithms, and the use of genetic algorithms which are implemented to produce the optimized structures studied here. Section 4 details how the optimization process is practically implemented, how the structure is modelled and the computing strategy used. Section 5 details the generation of 10 optimized structure candidates, generated for three different optimization criteria and briefly discussed the optimized designs obtained. The robustness of these designs is investigated in section 6. The conclusions drawn from the work are in section 7.

\section{THE STRUCTURE}

The structure studied is shown in Figure 1, in which the co-ordinate units are in meters (after reference [3]). It is a lightweight cantilever structure comprising 40 beams of lengths 1 and $1.414 \mathrm{~m}$. The structure and its vibration is considered in two dimensions; motion is only considered in the $x-y$ plane. A typical application for such a structure is that of an antenna boom arm for use on a satellite. In this scenario, the aim is to reduce the vibration transmission from the base of the structure to the rightmost beam, labelled beam 40 , where the antenna would be mounted in practice.

The physical properties of the beams used in the model are given: the bending rigidity, $E I$, is $69.80 \mathrm{MN}$; the axial rigidity, $E A$, is $12.86 \mathrm{kN} \mathrm{m}^{2}$; the mass per unit length is $2.74 \mathrm{~kg} \mathrm{~m}^{-1}$. This is found to correspond to an aluminium beam of approximate rectangular cross-sectional dimensions $50 \mathrm{~mm} \times 25 \mathrm{~mm}$, with the longer dimension in the $x-y$ plane. A proportional damping model is used for the beams [6] and therefore all the modes of the uncoupled beams have the same bandwidth. A value of $20 \mathrm{~s}^{-1}$ is used. This choice of damping parameter value is not untypical and was chosen so that a modal response was clearly evident in the structure's response, but not so low such that large resonant peaks caused noise problems due to a large dynamic range.

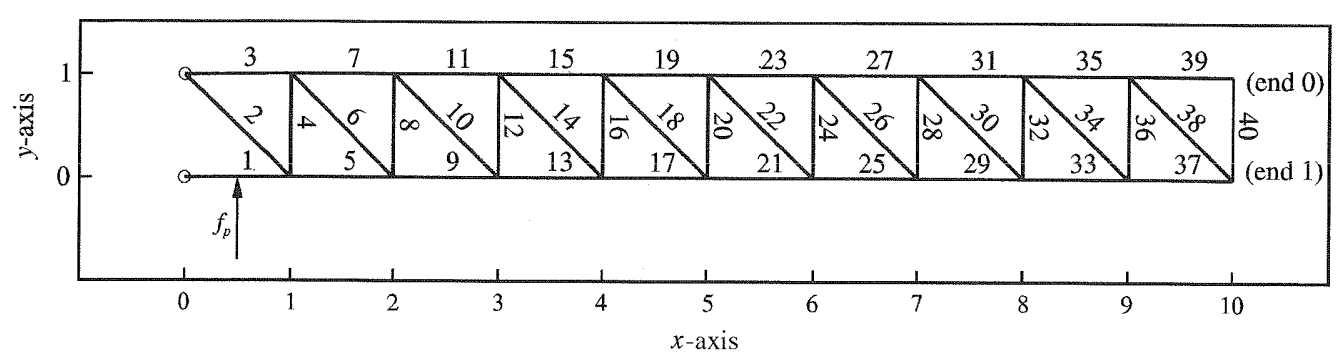

Figure 1. Unoptimized structure showing global co-ordinates, primary input force and beam numbering. 
The flexural vibration of each beam is modelled using the Euler-Bernoulli model [7] and a longitudinal vibration model, both using modal series summations. The response of the structure is analyzed by studying the coupling between all the individual beams. This is achieved using a receptance analysis [7] in which the unknown displacements, forces and moments are solved for each beam end when driven by the external force inputs. This is achieved at all the beam ends at each joint by equating the displacements and rotations, and summing the net forces and moments to zero. From this analysis the power transmitted into or out of each beam end and externally applied forces can be calculated. This process is described in detail in section 4.

The forces and moments at each beam end are solved by incorporating the individual beam receptance relationships into a global receptance matrix, and calculating the inverse to this matrix, which then enables the displacements and rotations to be evaluated. For the structure analyzed here the size of this matrix is approximately $170 \times 170$ elements. The optimization parameter is normally an average value across a frequency band, and hence the solution for each design scenario evaluated is computationally expensive. Moreover, extensive work with this class of problems shows them to be highly multipeaked in terms of the changes in their global vibration response to alteration in geometry as might be expected. The optimization becomes practically realizable with the use of evolutionary algorithms.

\section{DESIGN OPTIMIZATION}

\subsection{EVOLUTIONARY ALGORITHM OPTIMIZATION}

Optimization is a design process in which design is optimized by minimizing (or maximizing) one or more parameters defining relevant aspects of performance. The parameter, known as the objective function, is optimized by adjusting certain other parameters, optimization variables, under the control of the optimization algorithm. During optimization the algorithm assigns new sets of optimization variables from the combinations of the current sets under some strategy in which, on an average, those having better values of objective function are more likely to survive (in rather simplistic terms). The domain covered by all the possible combinations of optimization variables is known as the search space. Traditional methods of design optimization often rely on gradient-based methods and where the search space is continuous and uni-modal (and convex) they can perform very efficiently. In designs where the search space is multi-modal and contains many sub-optima these methods can result in a sub-optimal choices as only the local neighbouring search space is explored. Additionally, if the search space is discontinuous then such methods cannot be used.

Since first being reported by Holland [8], evolutionary algorithms have emerged in recent years as being an effective and efficient optimization technique. They are a stochastic-based class of optimizers, that are not random searches but have random elements in their algorithms that provide diversity to the search enabling the entire search space to be available for possible search progression, from any one 
point within the space. Evolutionary algorithms are best suited to finding optimal solutions to highly combinatorial problems, where an exhaustive search is not practicable or where the surface to be evaluated is multi-modal. Here the multiple local maxima would deceive conventional gradient searching algorithms.

Evolutionary algorithm is a generic term for a number of guided random search methods, of which the most popular two are genetic algorithms and simulated annealing. In general, genetic algorithms sample the search space more diversely; however simulated annealing has the advantage of requiring less computational effort. The choice, implementation and success of evolutionary algorithms is dependent upon the application. Keane [9] shows how different evolutionary algorithms sample a "difficult" search space. The value of combining algorithms, like genetic algorithms and simulated annealing, is also shown. In the optimization application considered, genetic algorithms have been used to enable comparison and verification of the work done by Keane [3].

When applying evolutionary algorithms it can never normally be established whether the true global optimal solution has been found. Repeated application of the algorithm yields near-optimal solutions and in most cases these out perform the existing design, and seeking the true globally optimum design is often not a necessity. In many applications there is little difference between the near-optimal and globally optimal solutions.

\subsection{GENETIC ALGORITHMS}

Genetic algorithms model the Darwinian evolution of nature. The design problem is specified in terms of a set of parameters which are to be optimized and an objective function (or fitness function) which enables the fitness of each solution (i.e., the relative performance) to be evaluated. The value of this function is maximized (or minimized dependent on the specific problem) in order to achieve the optimized design, defined by the design parameters or optimization variables. The optimization variables are coded in the form of a string which is usually a binary representation known as a "chromosome". Each different chromosome corresponds to a unique set of parameter values.

The genetic algorithm is initialized with a pool of chromosomes. The next generation is then achieved by the key operations: selection, crossover and mutation. A number of the previous generation's chromosomes are selected such that those with greater fitnesses have a higher probability of selection. Some of these chromosomes are then "mated" in pairs; two mating chromosomes swap information beyond a crossover point which is randomly selected, and two offsprings thus result. The new generation is made up of a proportion of newly formed and existing chromosomes from the previous generation. The last operation, mutation, is a random bit change in a chromosome with a small probability. This provides random diversity in the evolution and helps to prevent premature convergence before too little evolutionary experience has been gained.

The average fitness of the generation successively increases and the process is halted after a number of generations by a suitable convergence criterion. Normally, the best solution encountered through the entire optimization is taken as the result. 
In the elitist strategy, the best-so-far solution is guaranteed to survive into the next generation. Goldberg [10] analyzed the underlying nature of the algorithm using schemata to represent common patterns within the strings (a subset of the search space). The schema with higher fitness experience, on an average exponentially increasing trials in subsequent generations. The basis towards particular schema, representing a number of solutions, implies an implicit parallelism so that the search space is sampled diversely and efficiently.

A more complete description of genetic algorithms is available from Reference 10 , a well referenced book which provides good introduction to the early use of genetic algorithms, or from more recent and much more comprehensive texts [11].

\subsection{APPLICATION OF GENETIC ALGORITHMS TO THE STRUCTURE}

Following the optimization scenario in reference [3], genetic algorithms were applied to adjust the geometry of the structure shown in Figure 1 to minimize its vibration transmission. The relative positions between the fixed joints at co-ordinates $(0,0)$ and $(0,1)$ and the end joints at $(10,0)$ and $(10,1)$ are to remain unchanged. The joints at $(0,0)$ and $(0,1)$ are hinged while all the other joints are fixed (as if welded, for example). There are 18 joint positions to be determined by the optimization. Each joint position is defined by its $x$ and $y$ co-ordinates, making 36 optimization variables in total. Each variable is coded as a 16 bit string, this representation is scaled linearly between its limits. In this case the limits are $\pm 0.25 \mathrm{~m}$ about each nominal joint position. This was the maximum freedom reported by Keane [3]. This gives a precision of about $10 \mu \mathrm{m}$, which while practically unrealistic was retained for consistency with previous work [3]. Eight bits would provide a sufficient precision (of $2 \mathrm{~mm}$ ); however this does not significantly affect the operation or the speed of the algorithm. The binary strings are concatenated to form one long "chromosome" which is the unit of population for the genetic algorithm optimization. The $x$ and $y$ co-ordinates for the 18 joints free to move thus form 36 optimization variables. This scenario is then the same used in the optimization study performed in reference [3].

\section{IMPLEMENTATION OF STRUCTURE MODEL AND OPTIMIZATION}

\subsection{MODELLING THE STRUCTURE}

The overall structure is modelled by the receptance method (originally developed by Bishop and Johnston [7], and applied more specifically to this application by Shankar and Keane [12]) whereby the displacement response to at a force input is studied for each beam. The analysis considers forces in three planes: axial, transverse and rotational (moments) relative to each beam, and solves for both axial and transverse displacements and rotations. In solving for the overall response of the structure these are calculated for all beam ends and also the points where external forces are applied. The response of the whole structure; the application of a force at one point on a beam to a response on any other, is achieved by considering the end conditions of the beams which are joined together. 
This method has been used previously by Shankar and Keane and is detailed in reference [12]. Using matrix representation each beam is represented by a $3 \times 3$ matrix containing Green functions which describe the relationship between forces applied at a point on a beam to its response at another point, in three degrees of freedom. From the receptance matrices describing individual beams a global matrix is formed, and is determined by the topology of the structure. The matrix is then solved by equating the displacements at each joint, and by equating the net forces at each joint to zero. (For hinged joints, which only exist at the base of the structure, net moments are permitted). The solution is achieved by finding the inverse of the global receptance matrix, which then enables all the force components for each beam end to be found. The displacements and rotations can then be found by substituting the forces back into the receptance equations. The displacements and rotations at the end of the beam (which is the optimization subject) are converted to velocities. The power transmitted into or out of each beam end then enables the energy level in a beam to be calculated (which is equal to the net power dissipated in the beam divided by the damping). This is the value of the objective function and used by the optimization, for each particular joint set of positions evaluated. The modelling of the structure was achieved using code previously used by Shankar and Keane [12], who also validated the model with a comparison to a finite element model. By the use of the energy level and not the velocity at a single point, the optimization results are less specific, and allow a general measure of the reduction in vibration of a beam to be achieved and not solely at a particular, and perhaps arbitrarily chosen, point.

\section{GENERATION OF OPTIMAL DESIGNS}

Optimized designs of the structure shown in Figure 1 were sought, in order to minimize the vibrational energy transmission to the rightmost beam (labelled beam 40) from the structure base using three objective functions. The objective functions evaluated the energy in the end beam, (i) at a single frequency $(185 \mathrm{~Hz}$ ), (ii) as an average over a $20 \mathrm{~Hz}$ bandwidth $(175-195 \mathrm{~Hz}$ ), and (iii) as an average over a $100 \mathrm{~Hz}$ bandwidth $(150-250 \mathrm{~Hz})$. These are referred to as single-frequency, narrow-band and broadband optimizations. The energy level responses were produced by using a harmonic transverse input force applied to a beam at coordinates $(0.5,0)$. This force has an arbitrary value of $1 \mathrm{~N}$ at each frequency, and the response is calculated in frequency steps of $5 \mathrm{~Hz}$.

In order to gain an appreciation for the complexity of the optimization, Figure 2 shows a contour plot giving the variation of the objective function with both the $x$ and $y$ co-ordinate allowed under the optimization limits for joint 8 at $(4,1)$. Here the true multi-modality of the problem is evident. This, however, is with the remaining optimization variables at their nominal position whilst in the optimization process the surface shown here would also vary as other variables are adjusted.

For each objective function type, 10 optimized design candidates were produced and these were uniquely achieved by discarding a different number of random number of samples before commencing the optimization process. The resulting structures are labelled with suffixes " $A$ " to "_ J". For each optimized candidate the 


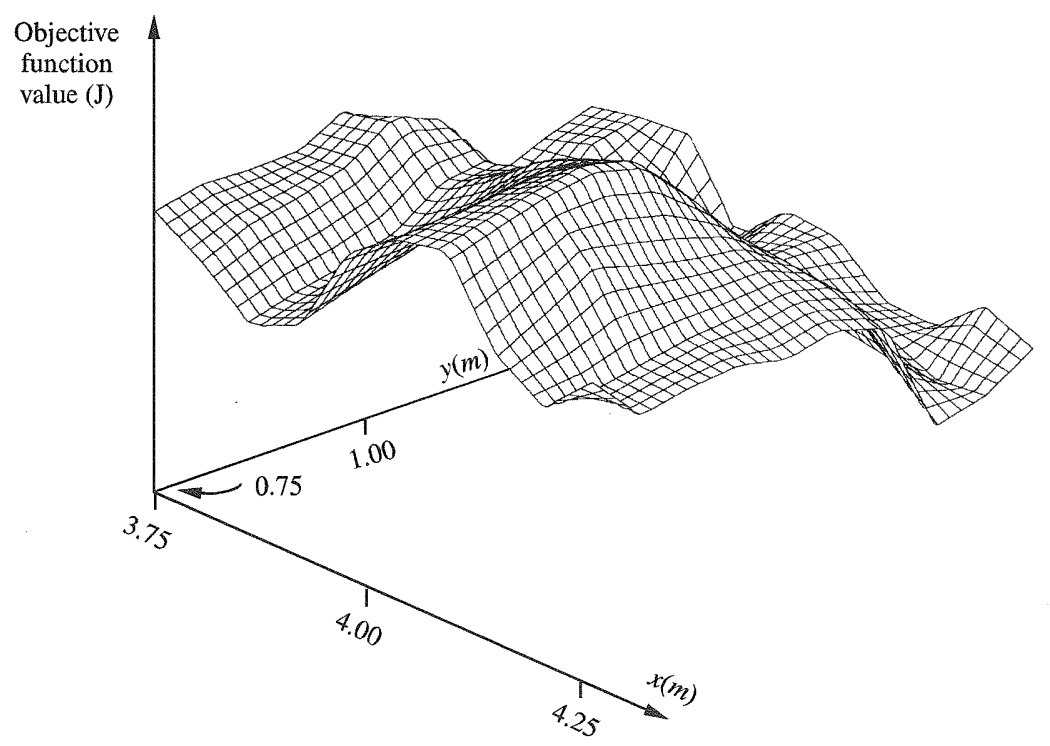

Figure 2. Contour plot of objective function against adjustment of joint 8 within the optimization limits.

structure geometry is used to calculate the energy response for a wider bandwidth $(50-350 \mathrm{~Hz}$ in $5 \mathrm{~Hz}$ steps, subsequently referred to as the wide-band response) to enable the effect of optimization to the regions outside of frequency band considered by the optimization to be seen.

The size of the optimization problem can be appreciated from the fact that there are 36 variables, each represented by a 16-bit number, giving $65536^{36}$ (of the order of $10^{173}$ ) possible combinations. Even if 8-bit representation had been used as previously discussed, this still would yield a search space of the order of $10^{85}$ possible combinations. If the objective function took a mere $1 \mathrm{~ms}$ to evaluate (each objective function here takes about $85 \mathrm{~s}$ ) then in the order of $10^{74}$ years would be needed to exhaustively explore the search space. Bäck [13] uses for comparison quantities relating to the universe, stating $10^{80}$ as being the number of stable elementary particles in the universe. The genetic algorithm calculates each of the optimized candidate structures by evaluating a maximum of 1000 structure designs (which were realized as five generations each of population 200) for the single-frequency and narrow-band cases, and a maximum of 4500 structure designs (15 generations of 300 ) for the broadband case. Although it is not known whether the global optimum is contained within the 10 candidates produced, substantial improvements are found in the performance for each case. A similar magnitude of performance improvement was obtained for each candidate.

\subsection{SINGLE-FREQUENCY OPTIMIZATION}

The optimization was first performed using an objective function equal to the energy level in the end beam at a single frequency, $185 \mathrm{~Hz}$. Figure 3 shows the best 


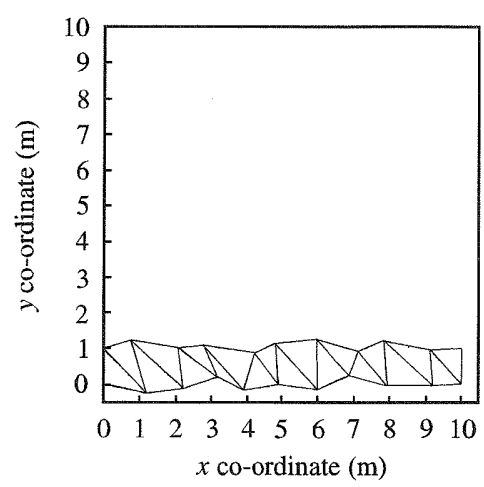

Single frequency $(185 \mathrm{~Hz})$ optimized structure SF_E

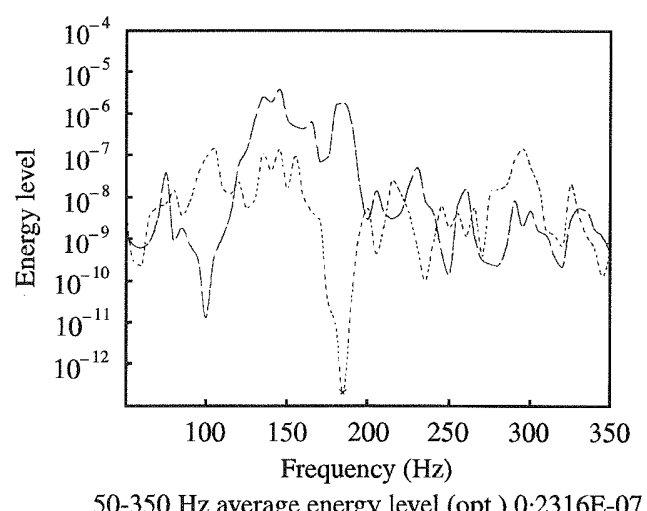

50-350 Hz average energy level (opt.) $0 \cdot 2316 \mathrm{E}-07$

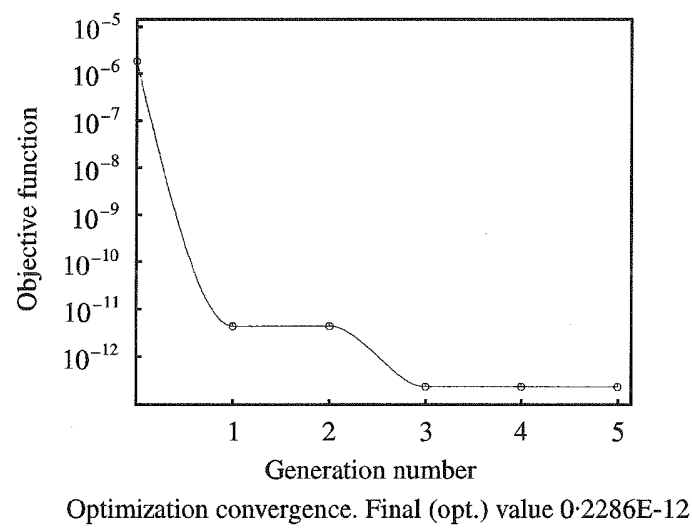

Figure 3. The best candidate structure and results for the Single-frequency objective function. (Energy level: optimization window $x \times$; ------ optimized response; - - - unoptimized response.)

candidate geometry achieved from the 10 optimization processes performed. The optimized structure topology is shown in the top-left-hand corner, the frequency response in the top-right-hand corner and the history of the objective function against generation at the bottom, which shows the value of the best objective function achieved after each generation. The final linear value of the objective function is also stated. The frequency response for the unoptimized structure is shown by a dashed line and for the optimized structure as dotted. The actual frequency value used for the objective function is denoted by a cross. The numerical results for all 10 candidates are summarized in Table 1, and the reductions in the objective function and wide-band average energy level achieved are shown in decibels relative to the unoptimized structure.

As the evaluated frequency is in a dominant resonant peak in the nominal response, it is not surprising that substantial gains can be achieved in minimizing the objective function. Since the peaks in the frequency response are likely to occur due to the cumulative effect of individual system resonances such resonances are often sensitive. To diminish the resonance response is therefore relatively easily achievable, but to reduce the response further requires that the conditions occurring at this frequency become destructive. Here the reductions achieved in the objective function range from $53 \cdot 0 \mathrm{~dB}$ (structure $\mathrm{SF}_{-} \mathrm{I}$ ) to $69 \cdot 2 \mathrm{~dB}$ (structure 
TABLE 1

Results summary for the single-frequency objective function

\begin{tabular}{|c|c|c|c|c|}
\hline $\begin{array}{c}\text { Rank } \\
\text {. }\end{array}$ & Structure & $\begin{array}{c}\text { Reduction in } \\
\text { objective } \\
\text { function }(185 \mathrm{~Hz}) \\
\text { (dB) }\end{array}$ & $\begin{array}{l}\text { Reduction in } \\
\text { wide-band average } \\
\text { energy level } \\
(50-350 \mathrm{~Hz}) \\
\text { (dB) }\end{array}$ & $\begin{array}{c}95 \% \text { probable } \\
\text { minimum reduction } \\
\text { in perturbed objective } \\
\text { function }(\mathrm{dB})\end{array}$ \\
\hline- & unopt & 0 & 0 & - \\
\hline 1 & $\mathrm{SF}_{-} \mathrm{E}$ & $69 \cdot 2$ & $10 \cdot 7$ & $56 \cdot 5$ \\
\hline 2 & $S_{F} \_D$ & $67 \cdot 3$ & $11 \cdot 0$ & $39 \cdot 6$ \\
\hline 3 & SF_A & $63 \cdot 9$ & $9 \cdot 8$ & $60 \cdot 2$ \\
\hline 4 & SF_G & $62 \cdot 4$ & $6 \cdot 4$ & $58 \cdot 2$ \\
\hline 5 & $\mathrm{SF}_{-} J$ & $60 \cdot 9$ & $4 \cdot 9$ & $55 \cdot 6$ \\
\hline 6 & $S F \_F$ & $60 \cdot 4$ & $7 \cdot 6$ & $55 \cdot 3$ \\
\hline 7 & SF_C & $60 \cdot 3$ & $6 \cdot 5$ & $39 \cdot 1$ \\
\hline 8 & SF_H & $58 \cdot 8$ & $5 \cdot 8$ & $50 \cdot 7$ \\
\hline 9 & $\mathrm{SF}_{-} \mathrm{B}$ & $56 \cdot 5$ & $8 \cdot 0$ & $39 \cdot 1$ \\
\hline 10 & SF_I & $53 \cdot 0$ & $7 \cdot 4$ & $46 \cdot 3$ \\
\hline
\end{tabular}

SF_E). The reduction of the wide-band response is more consistent, ranging from $4 \cdot 7$ (structure $\mathrm{SF}_{-} \mathrm{J}$ ) to $11 \mathrm{~dB}$ (structure SF_D) .

\subsection{NARROW-BAND OPTIMIZATION}

This optimization was performed with an objective function which was the average of the energy level of five frequencies, $175-195 \mathrm{~Hz}$ in $5 \mathrm{~Hz}$ steps. The candidate geometries which gave the best performance produced by 10 narrow-band optimizations are shown in Figure 4. The frequency range used by the objective function is shown as a solid section on the dotted response, with crosses additionally denoting the actual frequency points used. The numerical results for all 10 candidates are summarized in Table 2.

All of the optimized structures achieved reductions in the objective function with a range of $38.1 \mathrm{~dB}$ to $47.5 \mathrm{~dB}$. The average wide-band response is also reduced from $5 \cdot 2 \mathrm{~dB}$ to $12 \cdot 5 \mathrm{~dB}$. Even though only a small part of the frequency range was considered during the optimization, it is not surprising that this still achieves global reductions in the wide-band response, since the optimization window covers a dominant resonance peak in the response of the structure. After optimization the objective function is found to be an insignificant component of the wide-band response average. The structure achieving the best objective function reduction is structure $N_{-} B$, followed closely by structure $N_{-} G$. The best wide-band frequency average reductions are found in structures $N_{-} J$ and $N_{-} I$ respectively.

\subsection{BROADBAND OPTIMIZATION}

This optimization was performed with an objective function which was the average of the energy level in the end beam for 21 frequencies, $150-250 \mathrm{~Hz}$ in $5 \mathrm{~Hz}$ 


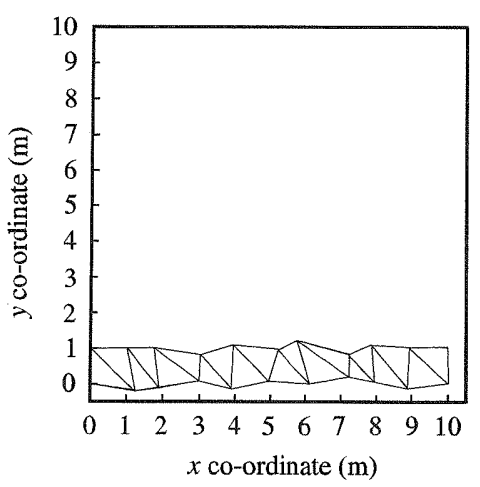

Narrow-band (175-195 Hz) optimized structure N_B

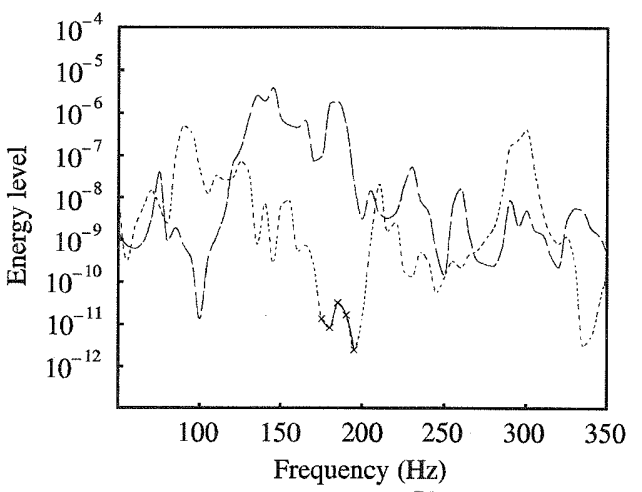

50-350 Hz average energy level (opt.) $0 \cdot 3829 \mathrm{E}-07$

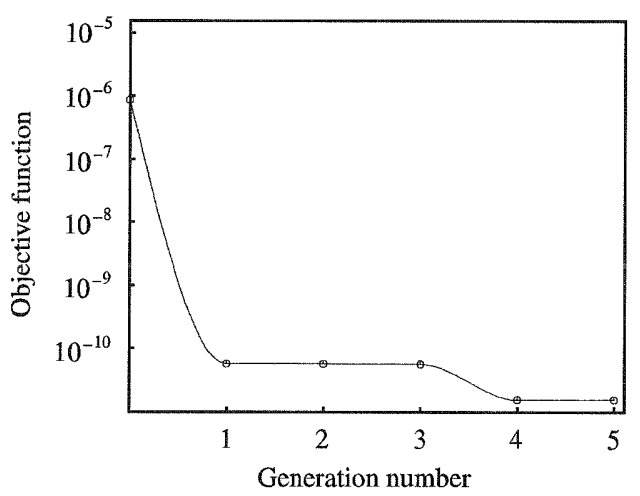

Optimization convergence. Final (opt.) value $0 \cdot 1564 \mathrm{E}-10$

Figure 4. The best candidate structure and results for the narrow-band objective function. (Energy level: optimization window $* x$; ----- optimized response; - - - unoptimized response.)

TABLE 2

Results summary for the narrow-band objective function

\begin{tabular}{|c|c|c|c|c|}
\hline Rank & Structure & $\begin{array}{c}\text { Reduction in } \\
\text { objective } \\
\text { function }(175-195 \mathrm{~Hz}) \\
(\mathrm{dB})\end{array}$ & $\begin{array}{l}\text { Reduction in } \\
\text { wide-band average } \\
\text { energy level } \\
(50-350 \mathrm{~Hz}) \\
\text { (dB) }\end{array}$ & $\begin{array}{c}95 \% \text { probable } \\
\text { minimum reduction } \\
\text { in perturbed objective } \\
\text { function }(\mathrm{dB})\end{array}$ \\
\hline - & unopt & 0 & 0 & - \\
\hline 1 & N_B & $47 \cdot 5$ & $8 \cdot 6$ & $42 \cdot 5$ \\
\hline 2 & N_G & $47 \cdot 1$ & $9 \cdot 8$ & $36 \cdot 6$ \\
\hline 3 & $N_{-} F$ & $45 \cdot 5$ & $7 \cdot 8$ & $42 \cdot 2$ \\
\hline 4 & $\mathrm{~N}_{-} \mathrm{J}$ & 44.9 & $12 \cdot 5$ & $41 \cdot 7$ \\
\hline 5 & N_A & $44 \cdot 4$ & $6 \cdot 7$ & $41 \cdot 3$ \\
\hline 6 & N_C & $43 \cdot 8$ & $8 \cdot 5$ & $39 \cdot 7$ \\
\hline 7 & N_H & $43 \cdot 2$ & $6 \cdot 6$ & $40 \cdot 2$ \\
\hline 8 & $\mathrm{~N}_{-} \mathrm{D}$ & $41 \cdot 8$ & $5 \cdot 2$ & $36 \cdot 3$ \\
\hline 9 & $\mathrm{~N}_{-} \mathrm{I}$ & $41 \cdot 5$ & $10 \cdot 7$ & $39 \cdot 0$ \\
\hline 10 & N_E & $38 \cdot 1$ & $9 \cdot 6$ & 31.9 \\
\hline
\end{tabular}




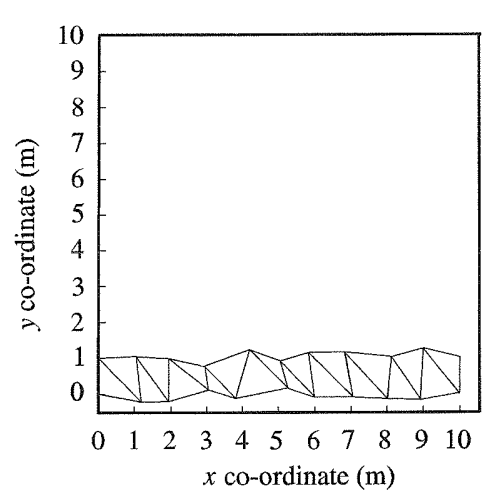

Broad-band (150-250 Hz) optimized structure B_E

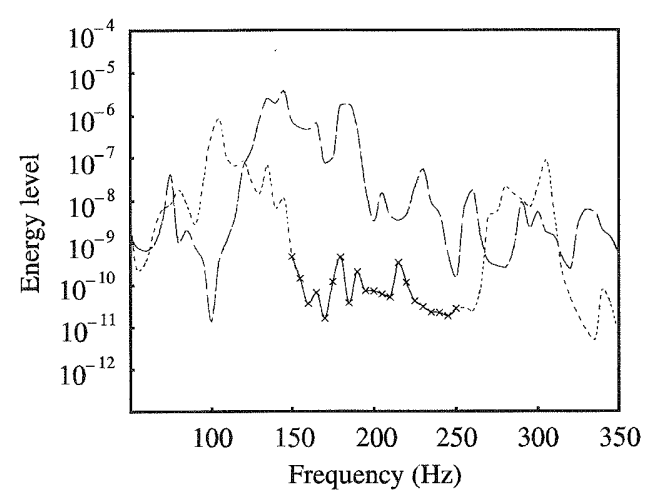

50-350 Hz average energy level (opt.) 0·3062E-07

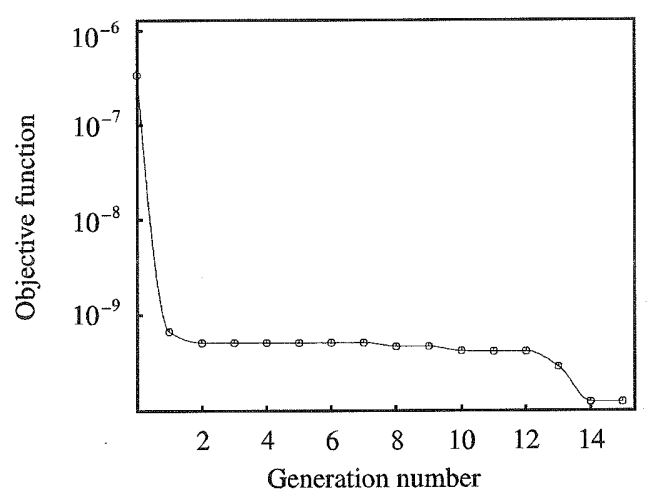

Optimization convergence. Final (opt.) value 0 $1198 \mathrm{E}-09$

Figure 5. The best candidate structure and results for the broadband objective function. (Energy level: optimization window $* *$; ----- optimized response; - - - unoptimized response.)

steps, as originally used in reference [3]. The best of the 10 optimized candidates produced is shown in Figure 5. Even though the parameters used were also used by Keane, the exact starting conditions used in his work could not be assured and therefore it is unlikely that any of the candidates produced here would be identical to any of those reported by Keane [3], which indeed is the case. However, the performance improvements achieved are of a similar magnitude. The numerical results for all 10 candidates are summarized in Table 3. It is noted incidentally that for this analysis each optimized candidate, evaluated on a high-performance hardware platform, took approximately 105 hours to produce.

The best reduction achieved in the objective function is $34.5 \mathrm{~dB}$ (structure $\mathrm{B}_{-} \mathrm{E}$ ) followed closely by $34 \cdot 1 \mathrm{~dB}$ (structure $\mathrm{B}_{-} \mathrm{F}$ ). However, these structures do not appear high in the ranking when ordered in terms of wide-band response reduction. In this respect, the best two structures are structures $B_{-} D$ and $B_{-} A$. The objective function covers a significant range of wide-band response than for the narrowband optimization, but the results show that this does not imply consistency in the ranking of the best structures in both objective function and wide-band response. 
TABLE 3

Results summary for the broadband objective function

\begin{tabular}{|c|c|c|c|c|}
\hline Rank & Structure & $\begin{array}{l}\text { Reduction in } \\
\text { objective } \\
\text { function } \\
(150-250 \mathrm{~Hz}) \\
\text { (dB) }\end{array}$ & $\begin{array}{c}\text { Reduction in } \\
\text { wide-band average } \\
\text { energy level } \\
(50-350 \mathrm{~Hz}) \\
\text { (dB) }\end{array}$ & $\begin{array}{c}95 \% \text { probable } \\
\text { minimum reduction } \\
\text { in perturbed objective } \\
\text { function }(\mathrm{dB})\end{array}$ \\
\hline - & unopt & 0 & 0 & - \\
\hline 1 & $\mathrm{~B}_{-} \mathrm{E}$ & $34 \cdot 5$ & $9 \cdot 5$ & 31.9 \\
\hline 2 & $\mathrm{~B}_{-} \mathrm{F}$ & $34 \cdot 1$ & $7 \cdot 2$ & $31 \cdot 1$ \\
\hline 3 & B_H & 33.9 & $9 \cdot 0$ & $31 \cdot 7$ \\
\hline 4 & $\mathrm{~B}_{-} \mathrm{B}$ & $33 \cdot 3$ & $12 \cdot 3$ & $27 \cdot 8$ \\
\hline 5 & B_C & $32 \cdot 8$ & $5 \cdot 3$ & $29 \cdot 2$ \\
\hline 6 & $\mathrm{~B}_{-} \mathrm{J}$ & $32 \cdot 3$ & 11.6 & $30 \cdot 9$ \\
\hline 7 & $\mathrm{~B}_{-} \mathrm{A}$ & $32 \cdot 3$ & $12 \cdot 7$ & $29 \cdot 8$ \\
\hline 8 & B_G & $32 \cdot 0$ & $8 \cdot 4$ & $30 \cdot 0$ \\
\hline 9 & B_D & $31 \cdot 6$ & $14 \cdot 2$ & $30 \cdot 2$ \\
\hline 10 & B_I & $31 \cdot 0$ & $8 \cdot 3$ & $26 \cdot 9$ \\
\hline
\end{tabular}

\subsection{DISCUSSION OF OPTIMIZATION RESULTS}

All optimization trials using objective functions were either based on an average of energy levels over a band of frequencies, or that using the energy level at a single frequency have produced substantial reductions in the objective functions. The mean and the maximum-to-minimum (max-to-min) ratio of the range of objective function values and the wide-band response values across the 10 candidates produced from each of the optimization cases are shown in Table 4. The wider the bandwidth of the objective function the smaller the mean reductions achieved across the 10 candidates; it can also be seen that the variation (max-to-min ratio) across the candidates in each optimization case decreases with bandwidth.

Each optimization seeks to achieve a reduction in the objective function, and the resulting wide-band response indicates that this is achieved at the expense of the response outside the optimization 'window'. This is shown by increases in the structural vibration transmission at some frequencies outside the objective function frequency range. Considering the reductions in the wide-band response for the optimized candidates, shown in Table 4, for all three optimization cases there are only small differences in the mean reductions and variations (max-to-min) in the wide-band reductions across each set of 10 candidates, indicating that in a global (i.e., wide band) sense the overall vibrational energy transmission achieved is similar. The mechanisms by which the minimization is achieved is thought to be by changing the many modes of vibration of the structure such that the energy transmission is affected in two ways. Firstly, the modes within the frequency band optimized are altered to move their resonant responses outside the frequency range of the objective function, or secondly, that the structural modes are aligned as to 
TABLE 4

Summary of optimization performance of the 10 structures optimized to reduce three objective functions

\begin{tabular}{ccrrr}
\hline \multicolumn{2}{c}{ Optimization case } & $\begin{array}{c}\text { Single } \\
\text { freq. }\end{array}$ & $\begin{array}{c}\text { Narrow } \\
\text { band }\end{array}$ & $\begin{array}{c}\text { Broad } \\
\text { band }\end{array}$ \\
\hline Objective function & Mean & $59 \cdot 0$ & $42 \cdot 9$ & $32 \cdot 6$ \\
$(\mathrm{~dB})$ & Max-to-min ratio & $16 \cdot 2$ & $9 \cdot 3$ & $3 \cdot 5$ \\
Wide-band response & Mean & $7 \cdot 4$ & $8 \cdot 1$ & $9 \cdot 1$ \\
$(\mathrm{~dB})$ & Max-to-min ratio & $6 \cdot 0$ & $7 \cdot 3$ & $8 \cdot 9$ \\
$95 \%$ probability limit & Mean & $44 \cdot 0$ & $37 \cdot 7$ & $29 \cdot 7$ \\
for objective function $(\mathrm{dB})$ & Max-to-min ratio & $21 \cdot 1$ & $10 \cdot 6$ & $5 \cdot 0$ \\
\hline
\end{tabular}

destructively interfere. The results presented here show that the average wide-band responses is only weakly dependent on the frequency band of the objective function.

In addition to being attributed to the successful application of genetic algorithms to find inherently better designs for the structures, the success is also probably attributed to the fact that the traditional periodic design is a particularly bad design with respect to the transmission of vibrational energy. The periodicity of the structure, whilst being favourable on aesthetic grounds allows similar frequency components, that would propagate relatively unimpeded through one bay section, through all the bay sections. The success in the optimized design candidates seems, in part, therefore to come from the non-repetitive nature of the geometry. Although the non-repetitive nature alone does not imply better performance (indeed, many such designs may be worse than the unoptimized structure), it is a common feature of all the optimized candidates.

\section{INVESTIGATION OF ROBUSTNESS}

The robustness of the performance to geometric perturbations was analyzed for each of the optimized structures obtained for the single-frequency case, for the narrow-band and broadband objective functions. Three hundred sets of joint perturbations were generated and applied to the joint positions of each structure and the objective function re-evaluated and recorded. Each set contained 18 records of random numbers distributed uniformly between -1 and 1 . Each record being formed of two numbers relate to the $x$ and $y$ co-ordinates for each joint. The same 300 sets of joint perturbations were applied to each structure. Each of the joint perturbation records were added to the joint co-ordinates for each structure in turn, suitably scaled. The change in the resulting objective function represents the sensitivity of the performance of the structure to small changes in the joint positions.

\subsection{ROBUSTNESS OF UNOPTIMIZED STRUCTURE}

A brief study of the unoptimized structure candidates performance due to geometric perturbations was conducted. The perturbed performance is presented 

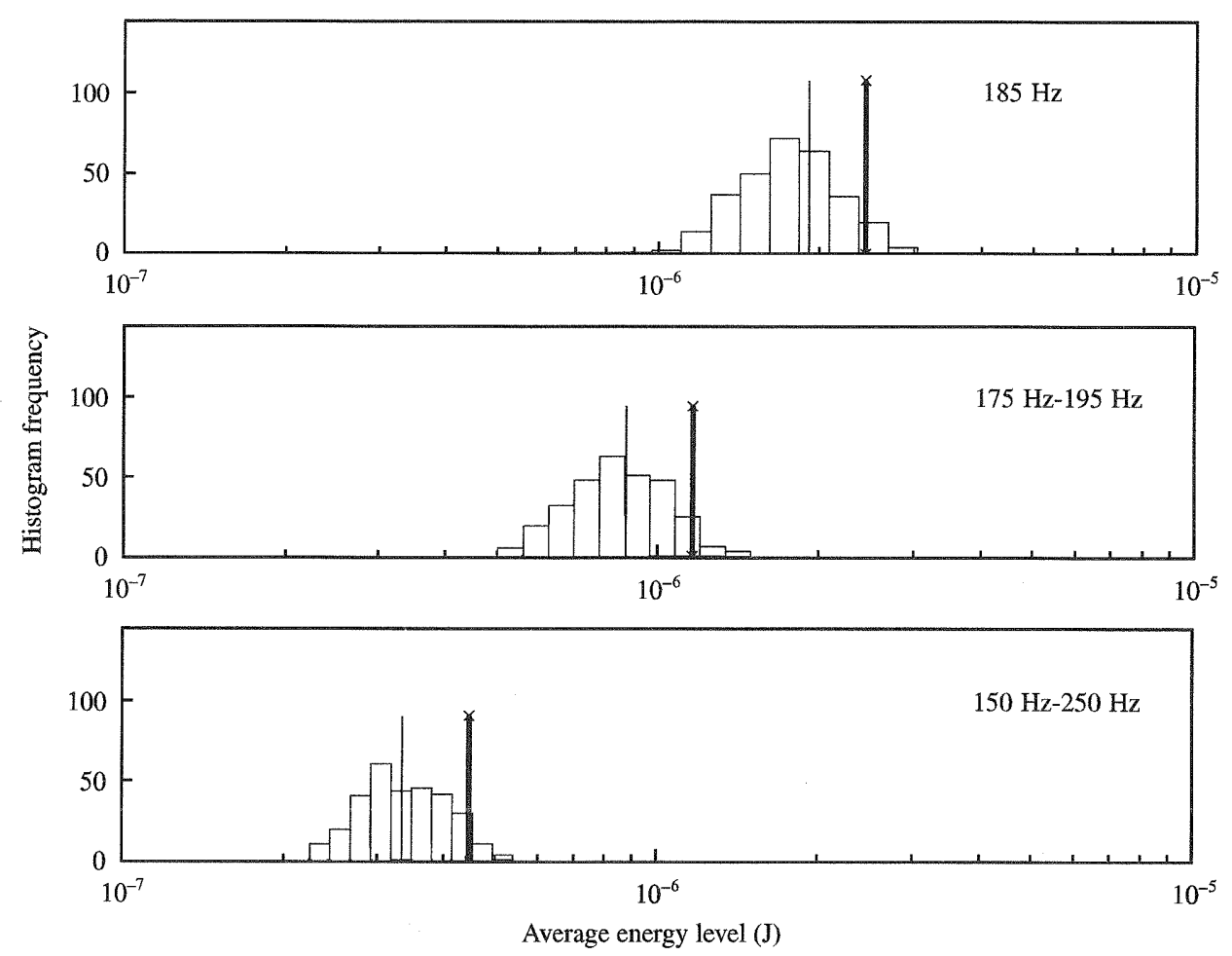

Figure 6. Statistical distribution of the unoptimized structure for all optimization bandwidths, for a perturbation scaling factor of $0 \cdot 01$.

for perturbation scaling factor of 0.01 for the random perturbations. This causes the maximum perturbation to each joint co-ordinate to be $\pm 10 \mathrm{~mm}$ in both $x$ - and $y$-axis. (The effect of this scaling factor is studied below for the narrow-band case.) The results are shown in Figure 6 by displaying the value of perturbed structure performance obtained using a histogram. For this and the following histograms the range is divided equally into 10 bars between the minimum and maximum values (when scaled logarithmically). The nominal (unperturbed) value is indicated by a thin solid line superimposed upon each histogram plot. The energy level averaged over the frequency bandwidth used for the optimization is higher for the single-frequency case as there is a strong resonant peak in the frequency response at this frequency; similarly the narrow bandwidth has a higher average than the broadband optimization bandwidth. The robustness is then determined by the spread of the results; the narrower it is, the more robust the structure. It is seen that for all the optimization bandwidths considered the robustness is similar. The solid line is the $95 \%$ performance probability limit, which determines the performance value for which, of the $95 \%$ of the applied perturbations the performance is better. This is discussed in detail in the following sections.

\subsection{ROBUSTNESS OF SINGLE-FREQUENCY OPTIMIZED STRUCTURES}

The robustness of the optimized structure candidates obtained using an objective function of the energy level at a single frequency was briefly studied. The perturbed 


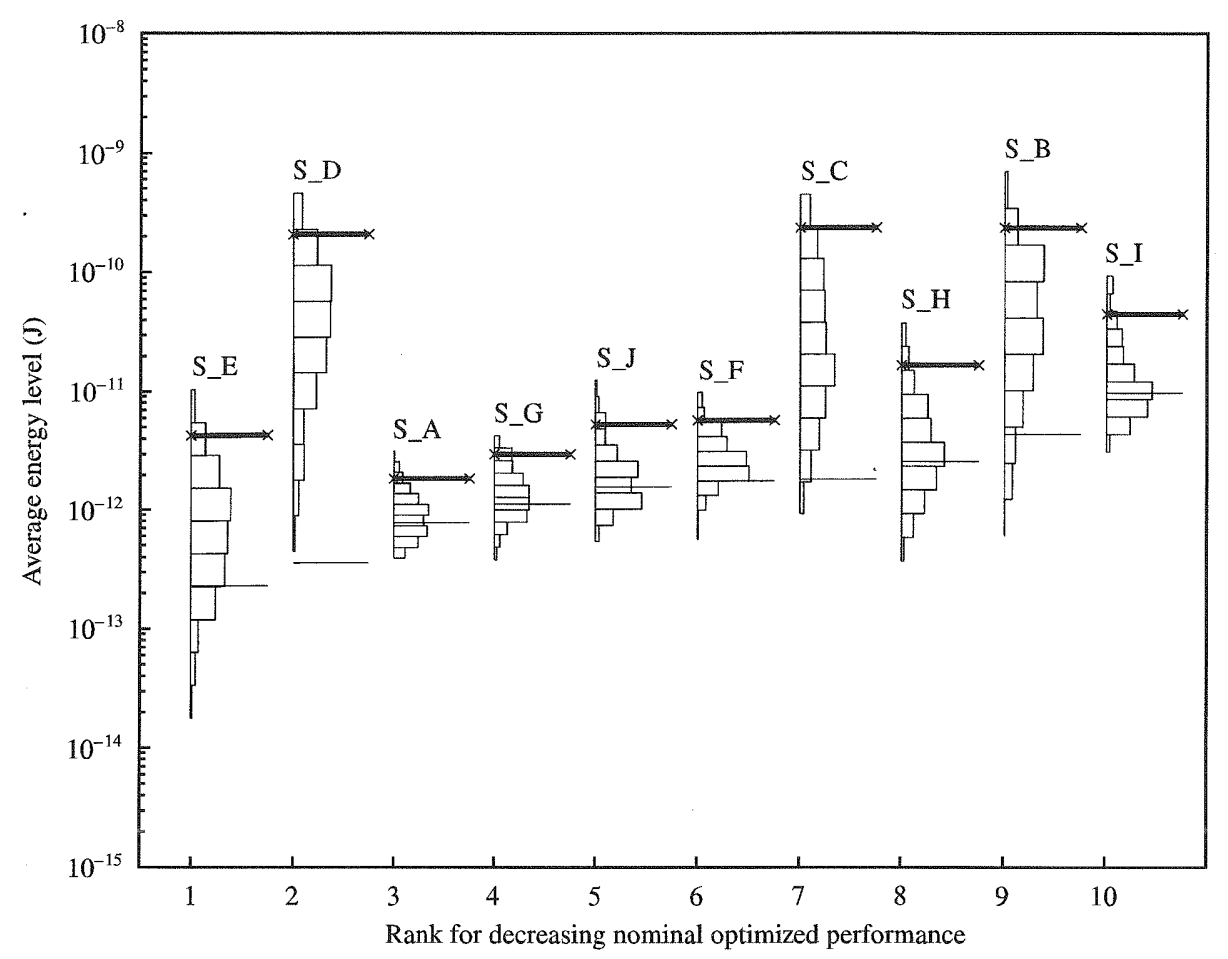

Figure 7. Statistical distribution and $95 \%$ probability limits for single-frequency optimized structures, for a perturbation scaling factor of $0 \cdot 01$.

performance is presented for a perturbation scaling factor of 0.01 for the random perturbations. This causes the maximum perturbation to each joint co-ordinate to be $\pm 10 \mathrm{~mm}$ in both $x$ - and $y$-axis, as previously for the unoptimized structure analysis.

The perturbed values of the objective function are displayed using a histogram to indicate the statistical spread about the nominal value (Figure 7). The nominal value is indicated by a thin solid line superimposed upon each histogram plot. The results for each structure are ranked in order of decreasing nominal performance for the structures. The results are listed in Table 1.

The robustness of each structure is indicated by the width of each statistical distribution. From this it can be said that, for example, S_E to S_D are less robust (i.e., more sensitive) than structures $S_{-} A$ and $S_{-} G$ to small perturbations in structure geometry. If solely the robustness of the structures were the paramount design goal then structure $\mathrm{S}_{-} \mathrm{A}$ is shown to have the best performance in this respect. However, even though this structure is more robust, its nominal (unperturbed) performance is not as good as structure $S_{-} E$.

It can be seen that the entire distribution of the performance for structure SF_D is worse than for the nominal structure for all perturbation cases (the nominal structure performance is not included in the distribution population). The size of the joint perturbations used here represents a generous manufacturing tolerance of 
$10 \mathrm{~mm}$. However, it is unlikely that the nominal performance of this structure would still be practically realizable. As shown below, where the size of the perturbations is studied, the perturbations used here are still representative of the robustness seen for smaller perturbations. Comparing the perturbed performance of the single-frequency optimized structure with that of the perturbed performance of the unoptimized structure in Figure 6, it is seen that in general the structures have a lower robustness after optimization. Only structures S_A and S_G have maintained a similar level robustness through optimization.

In most cases, the choice of the best optimized candidate will be determined by both the robustness and absolute performance of a structure. An overall measure of performance suggested here is the minimum reduction in perturbed objective function value with reference to the unoptimized structure which is achieved for $95 \%$ of the perturbations. Here the energy level is calculated for each structure's performance such that $95 \%$ of the perturbed performance is better than this limit. This criterion was applied to the perturbed performance results and is also shown in Figure 7 by a bold solid line. Using this criterion the best structure is seen to be structure $S_{-}$A, followed closely by structure $S_{-} G$ and then structures $S_{-} E_{-}, S_{-} J$ and $S_{-} F$. The performance $95 \%$ probability limits are also included in Table 1.

\subsection{ROBUSTNESS OF NARROW-BAND OPTIMIZED STRUCTURES}

The robustness of the narrow frequency band optimized structures was investigated using a scaling factor of 0.01 for the random joint co-ordinate perturbations up to $\pm 10 \mathrm{~mm}$. The nominal and perturbed performance of each structure is shown in Figure 8 with the $95 \%$ probability limits, following the convention of Figure 7. The results are also listed in Table 2.

On grounds of robustness alone structure $N_{-} I$ is the most robust structure; however its absolute performance is generally worse than most of the other candidates. This optimized structure is the only one whose robustness is comparable to the unoptimized structure, shown in Figure 6. Using the $95 \%$ probability limit the best structure is seen to be structure $N_{-} B$, which for this optimization case also happened to have the best nominal performance.

The choice of the maximum size of perturbations used above (determined by the perturbation scaling factor) was arbitrary to some extent, though it was chosen in order to represent a typical maximum manufacturing tolerance. In order to verify that the above results are not dependent on this scaling factor, and that there is some degree of "linearity" in the results against small changes in the scaling factor, two additional scaling factors were investigated. The results for scaling factors of 0.005 and 0.02 (perturbations of up to \pm 5 and $\pm 20 \mathrm{~mm}$ ) are shown in Figures 9 and 10, respectively, for the narrow-band optimized structures. In both cases, the absolute values defining the distribution are changed due to the different size of the applied perturbations.

The ranking of the $95 \%$ probability limits for a scaling factor of 0.005 across the structures is almost identical to those for $0 \cdot 01$, although the differences between structures $\mathrm{N}_{-} \mathrm{C}$ and $\mathrm{N}_{-} \mathrm{H}$ are becoming smaller. The performance limits for a scaling of 0.02 shows more radical changes in ordering than for those with 0.01 


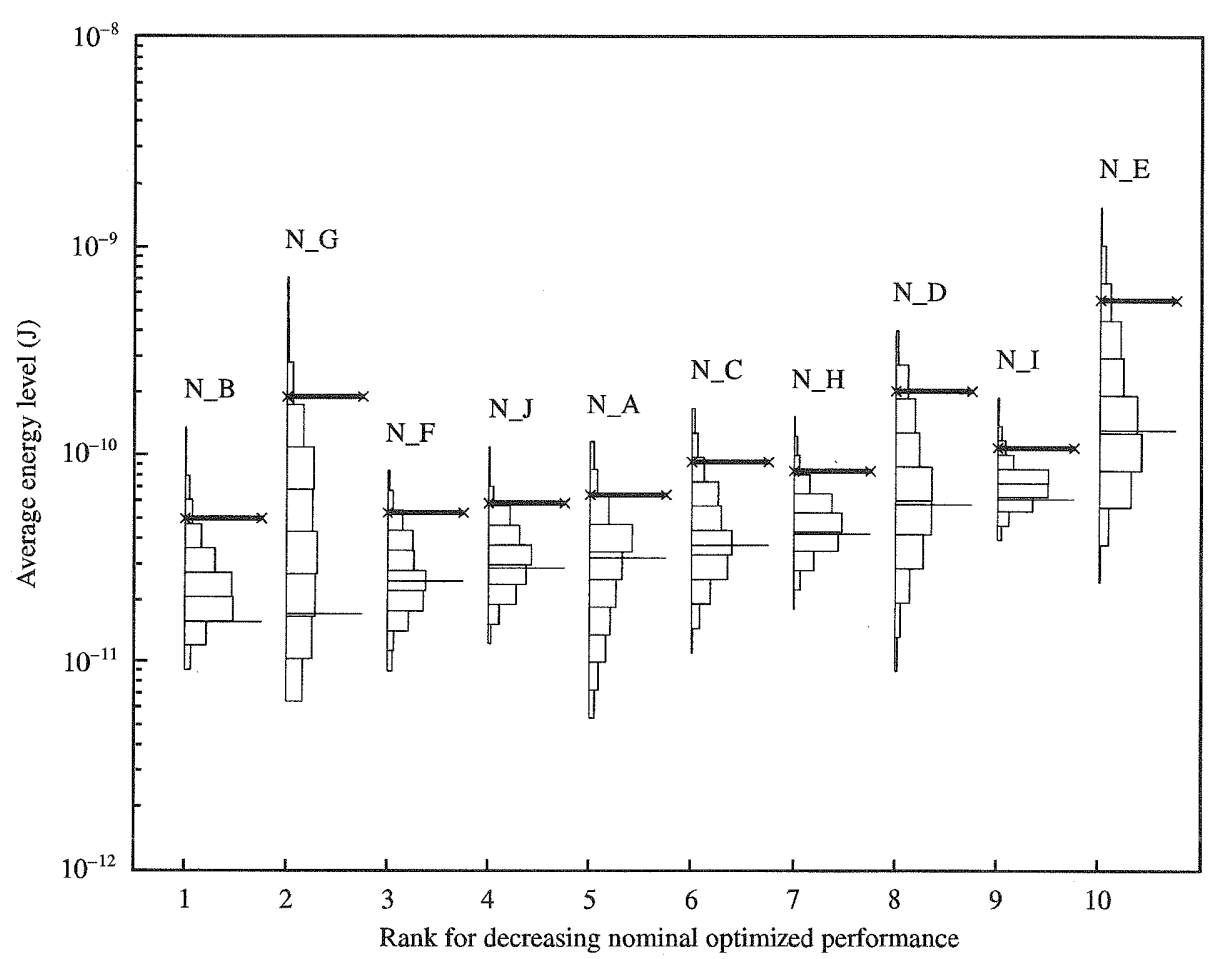

Figure 8 . Statistical distribution and $95 \%$ probability limits for narrowband optimized structures, for a perturbation scaling factor of $0 \cdot 01$.

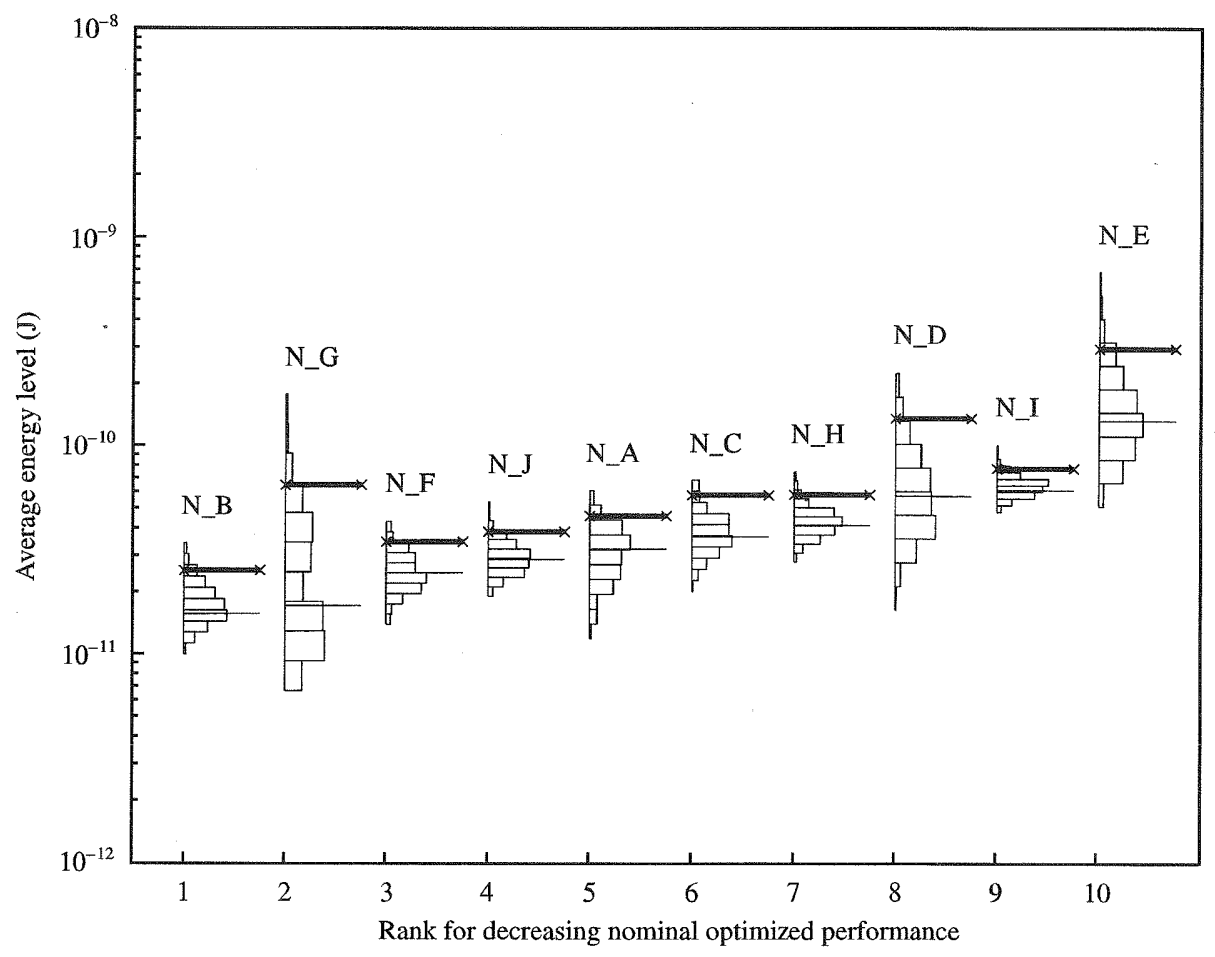

Figure 9. Statistical distribution and $95 \%$ probability limits for narrow-band optimized structures, for a perturbation scaling factor of 0-005. 


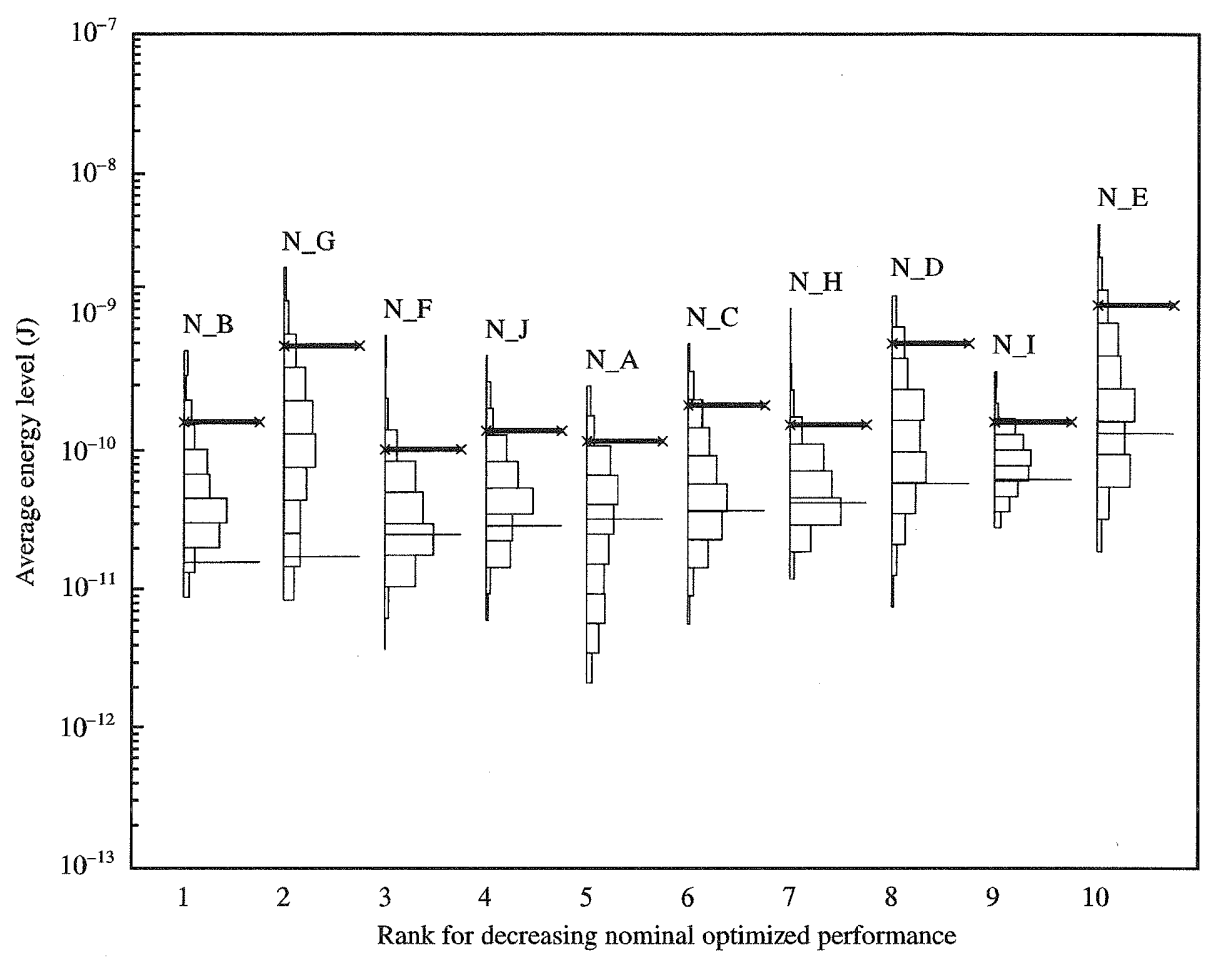

Figure 10. Statistical distribution and $95 \%$ probability limits for narrow-band optimized structures, for a perturbation scaling factor of 0.02 .

scaling. In particular, the original "best choice" performance of structure N_B has deteriorated. These results suggest that a scaling factor of 0.02 is too large to be considered a "small" perturbation for these structures, producing results strongly dependent upon the range of perturbation amplitudes.

\subsection{ROBUSTNESS OF BROADBAND OPTIMIZED STRUCTURES}

The robustness of the broadband optimized structures was investigated using perturbation scaling factor of 0.01 for the random perturbations. The nominal and perturbed performance of each structure is shown in Figure 11 with the 95\% probability limits following the convention of Figure 7. The results are also listed in Table 3.

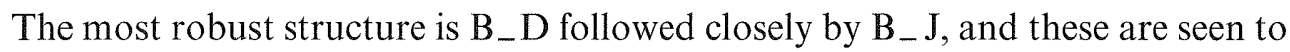
be more robust than the unoptimized structure, whose perturbation performance is shown in Figure 6. Although in general it is seen that the perturbations have less effect on the broadband optimized structures than the other two cases, the performance of some structures are clearly more sensitive to perturbations than others. This is shown by the reduced (vertical) baseline of the histograms. For the least robust structure $B_{-} B_{\text {this }}$ covers only one order of magnitude. Using the $95 \%$ probability limit the best structures are seen to be $B_{-} E$ and $B_{-} H_{\text {. }}$ 


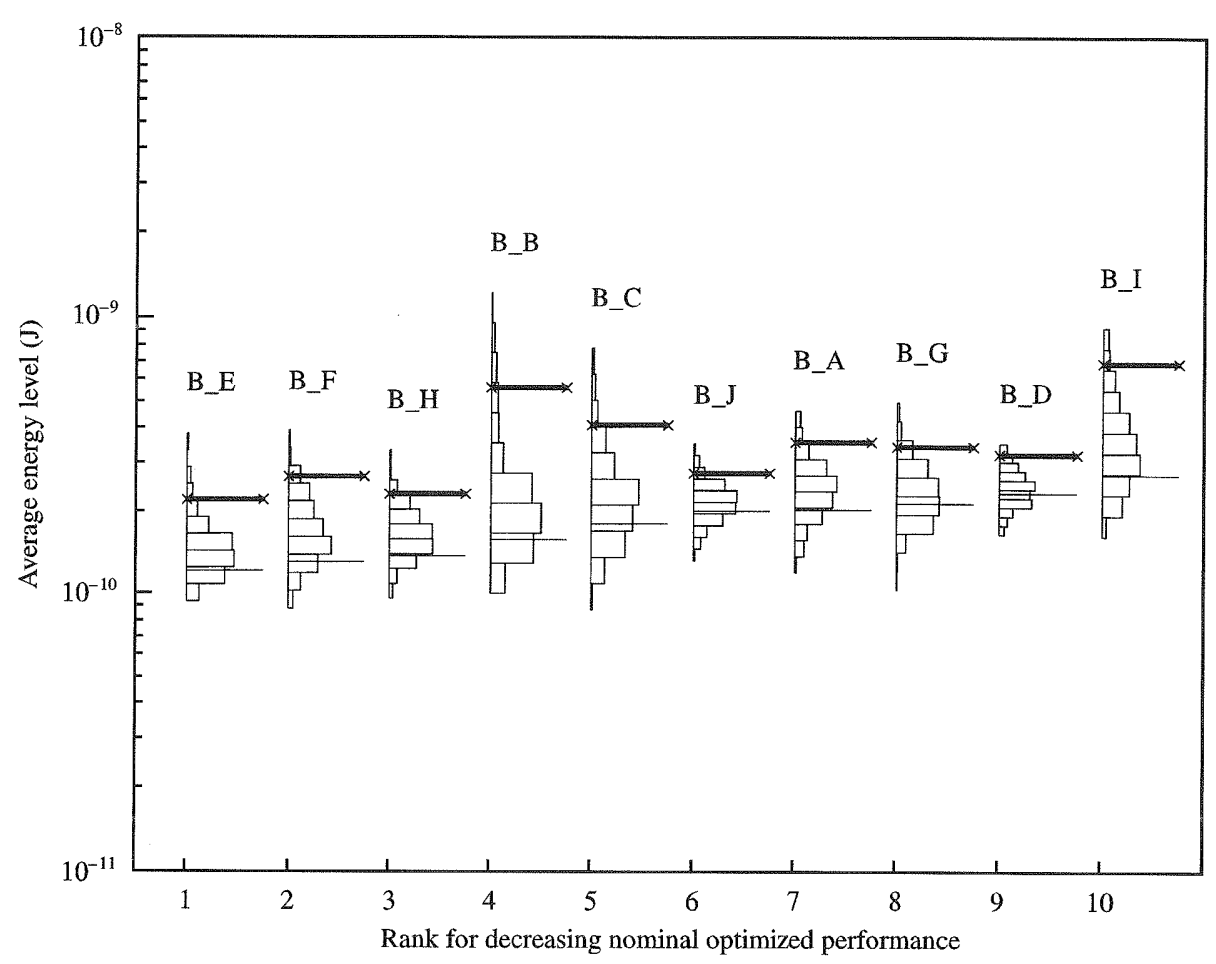

Figure 11. Statistical distribution and $95 \%$ probability limits for broadband optimized structures.

\subsection{COMPARISON OF ROBUSTNESS OF OPTIMIZED STRUCTURES ACHIEVED USING DIFFERENT OBJECTIVE FUNCTIONS}

Even though the optimized structures obtained for the single frequency, and both narrow and broadband objective functions are different, comparing the spread of the distributions for the candidates for each case an indication of the typical robustness inherently achieved with each type of objective function can be seen. A perturbation scaling of 0.01 (corresponding to perturbations up to $\pm 10 \mathrm{~mm}$ ) is common for each optimization case in Figures 7, 8 and 11. It is seen that for the same perturbations the wider the frequency band considered by the objective function the smaller the spread. For the single-frequency case, the spread of each candidate's distribution varies from three orders of magnitude to one order of magnitude; for the narrow-band case the spread varies from one to two orders of magnitude, and for the broadband case all the candidates variations fall approximately within one order of magnitude. Hence, it is seen that the wider the bandwidth of the objective function the more inherently robust the candidates produced by the optimization. However, only some of the candidates for the broadband optimization are shown to be more robust than the unoptimized structure.

The right-hand column of Tables 1-3 shows for each of the optimization cases the $95 \%$ probability limits for the individual optimized candidates. The mean and the maximum-to-minimum (max-to-min) ratio of the range of the $95 \%$ probability 
limits across the 10 candidates produced from each of the optimization cases are shown in Table 4 . The mean of the $95 \%$ probability limits is seen to decrease with increasing objective function bandwidth, whereas the min-to-max ratio is seen to decrease. This trend is similar to that for the corresponding objective function value results. This is explained by the fact that the $95 \%$ probability limit is a combined measure of the nominal performance and the robustness for each candidate. However, for the single-frequency case, the max-to-min ratio is noticeably greater due to the relative lack of robustness of the candidates produced from single-frequency optimization. In addition to the lack of robustness to geometric perturbations the single-frequency optimized structures will also be very sensitive to any change in excitation frequency. Hence, in practical terms, optimization to performance over a frequency bandwidth is always preferable.

The geometric perturbations of the structures above, and the results obtained from them, were obtained from the statistical performance due to an ensemble of uniformly distributed perturbations. If the distribution of the perturbations were to differ greatly from being uniform, then other candidate choices would have been preferable. In the absence of any information about the perturbation distribution the assumption of uniform distribution is prudent. If the distribution of the perturbations were known then this could have been applied in the analysis to produce more specifically relevant results.

\subsection{A NOTE ON THE ROBUSTNESS OF OPTIMIZED STRUCTURES ACHIEVED USING TRADITIONAL OPTIMIZATION METHODS}

The use of traditional optimization techniques whose strategy is to "climb" (or "descend") the "hill" (or "valley") nearest the initial position in the search space was briefly studied. The three techniques considered here are well known, and are: the Hook and Jeeves method [14], the Fletcher-Davidson-Powell method (FDP) [14] and the Broyden-Flecther-Goldfarb-Shanno method (BFGS) [15]. These techniques are commonly referred to as "hill climbers" (for both minimization and maximization problems). The implementations used are those as in the citations given. A general discussion of "hill climbers" may be found in either citation.

The results for the robustness analysis of the performance of the resulting structures are given for the broadband case only, and is shown in Figure 12. A perturbation scaling of 0.01 was used. Comparing these results with those of Figure 11, achieved using genetic algorithm optimization, it can be seen that all of the three designs have worse nominal performances. Additionally, it is also seen that the robustness of the optimized structure achieved using the Hook and Jeeves method is worse than those achieved using Genetic Algorithms; the robustness of the other two candidates is comparable. However, except for the BFGS optimized design, the nominal performance is outside the range of values of perturbed performance, and therefore is unlikely to be achieved in practice. Even for the BFGS optimized structure the nominal performance is very likely to be much worse, in practice. 


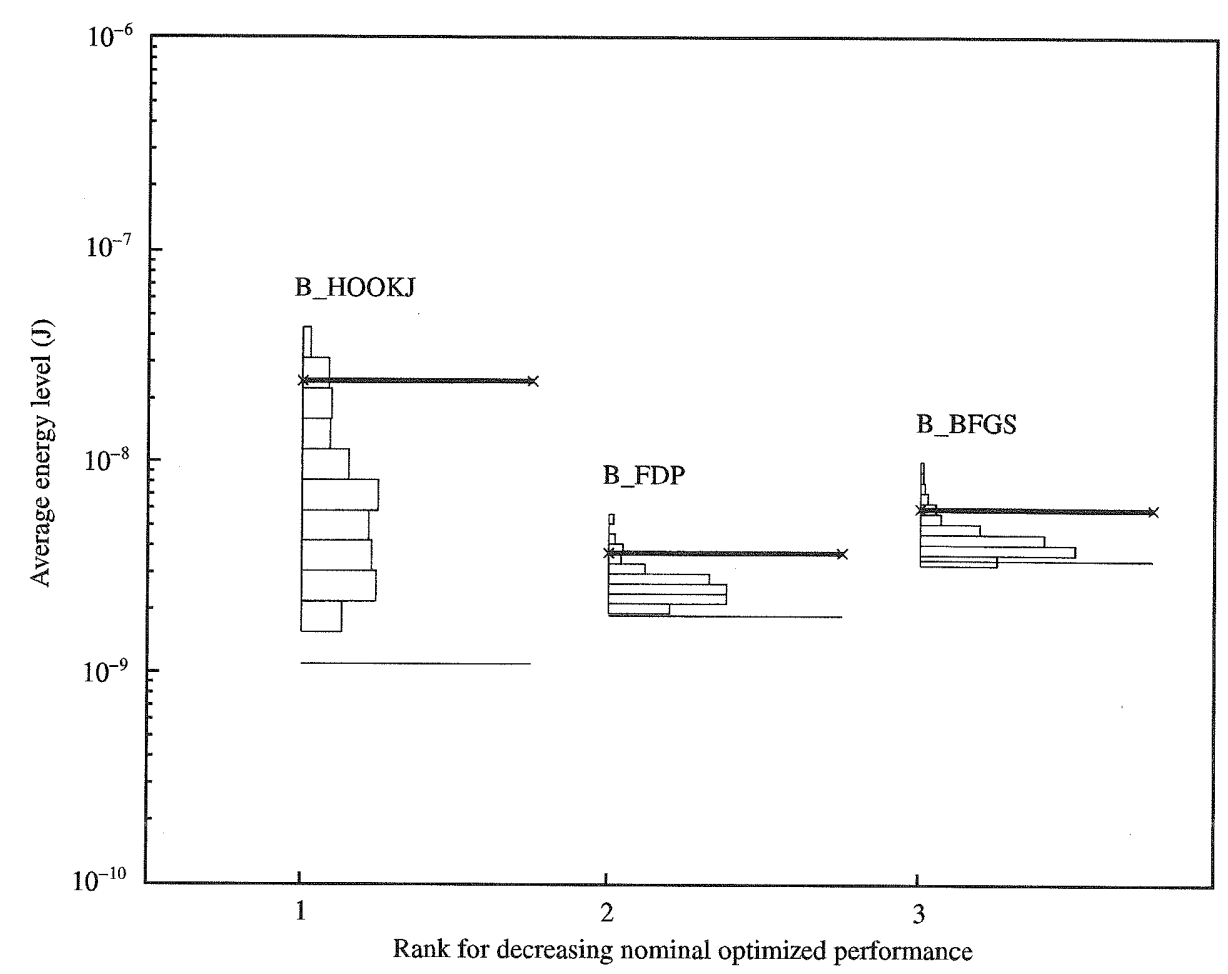

Figure 12. Statistical distribution and $95 \%$ probability limits for broadband optimization using Hook and Jeeves (B_HOOKJ), Fletcher-Davidon-Powell (B_FDP) and Broyden-Fletcher-Goldfarb-Shanno (B_BFGS) methods, for a perturbation scaling factor of 0.01 .

The inferiority of the nominal performance of the optimized designs in Figure 12 is due to the fact that the optimization methods used have descended into nearest "valley" in the search space. The better success achieved using genetic algorithms suggests that better optimal designs away from the locale of the initial search position. The unfeasibility of realizing the designs in practice is due to the strategy of the optimization techniques. The local minima in the search space are likely to be due to the cumulative destructive effect of many system anti-resonances, each of which is often sensitive to small changes in the system. Solutions found by such optimization techniques are often sensitive as hill-climbing techniques seek the very bottom of these often sharp "crevices" in the search space. This is not the strategy used by the genetic algorithms, and thus optimized structures tend to be more robust, and produce more practical solutions.

\section{CONCLUSIONS}

Optimization of an existing traditional design of a lightweight cantilever structure, to reduce the vibrational energy transmission from the base to end beam, was performed. The optimization criterion was based on three types of objective function, one using the energy level at a single frequency $(185 \mathrm{~Hz})$, one using an energy level average over a narrow band of frequencies $(175-195 \mathrm{~Hz})$, and one using 
an energy level average over a broad band of frequencies $(150-250 \mathrm{~Hz})$. Genetic algorithms were used to perform the optimization and 10 optimized structures were produced for each case.

The optimization process achieves significant reductions in the energy transmission in the frequency band. However, this appears to be partly at the expense of the transmission at other frequencies, as the average energy level over a wider bandwidth than that considered by the objective function appears to experience a similar reduction, almost independent of the objective function frequency band. It is suggested that this may either be due to the modes of vibration of the structure being altered by the optimization process to shift their energy transmission out of the frequency band considered in the optimization, or their alignment to destructively interfere.

The robustness (lack of sensitivity) of the optimized performance to small changes in the structure geometry of each structure candidate was analyzed by applying small perturbations to the positions of the non-extreme joint positions. By applying a common ensemble of random joint perturbations to each candidate the statistical distribution of the resulting performance change could be studied. It was found that some structures are more robust to such perturbations than others. Three different sizes of random joint perturbations were applied to the narrowband optimized candidates uniformly distributed between $\pm 5, \pm 10$ and $\pm 20 \mathrm{~mm}$. It was found that a similar ranking of results was obtained for perturbation between \pm 5 and $\pm 10 \mathrm{~mm}$. The ranking was not preserved perturbations up to $\pm 20 \mathrm{~mm}$ because, it is thought, they were no longer small compared to the size of the structure.

The choice of the best optimized candidate depends upon both the absolute value of the nominal (unperturbed) performance, and the robustness of the structures performance to perturbations. A criterion is suggested which defines the best candidate to be that whose performance is the best for $95 \%$ of all perturbations applied. This is used to indicate the statistical expectation of the structure performance. This criterion is applied to the candidates enabling the best candidate, in terms of both robustness and absolute performance, to be identified.

In general, it is found that the wider the response bandwidth considered by the objective function in the optimization process, the less the spread in nominal performance across the 10 candidates produced in each case and also the more robust the design candidates obtained. This is thought to be because the wider bandwidth will result in an objective function which is dependent on the combined effect of many modes and is therefore less sensitive to changes in any one particular mode.

The likelihood of producing impractical designs if traditional 'hill-climbing' optimization methods were used in place of genetic algorithm optimization was briefly demonstrated for the broad band case using three such well-known methods.

\section{ACKNOWLEDGMENT}

The first author acknowledges support in the form of a studentship from the Faculty of Engineering \& Applied Science at The University of Southampton. 


\section{REFERENCES}

1. C. R. Fuller, S. J. Elliott and P. A. Nelson 1996 Active Control of Vibration. London: Academic Press.

2. D. K. Anthony and S. J. Elliott Journal of Sound and Vibration. Robustness of optimal design solutions to reduce vibration transmission in a lightweight 2-D structure. Part II: application of active vibration control techniques (submitted).

3. A. J. Keane 1995 Journal of Sound and Vibration 185, 441-453. Passive vibration control via Unusual geometries- the application of genetic algorithm optimization to structural design.

4. A. J. Keane and A. P. Bright 1996. Journal of Sound and Vibration 190, 713-719. Passive vibration control via unusual geometries: experiments on model aerospace structures.

5. A. J. KeANE and S. M. Brown 1996 Proceedings of ACEDC '96 PEDC, University of Plymouth, U.K., 107-113. The design of a satellite boom with enhanced vibration performance using genetic algorithm techniques.

6. F. S. Tse, I. E. Morse and R. T. Hinkle 1978. Mechanical Vibrations. Theory and Applications. Boston, MA: Allyn and Bacon, Inc.

7. R. E. D. Bishop and D. C. Johnson 1960. The Mechanics of Vibration. Cambridge: Cambridge University Press.

8. J. H. Holland 1975 Adaptation in Natural and Artificial Systems: An Introductory Analysis with Applications to Biology, Control and Artificial Intelligence. Ann Arbor, MI: The University of Michigan Press.

9. A. J. KeAne 1995 Artificial Intelligence in Engineering 9, 75-83. Genetic algorithm optimization of multi-peak problems - studies in convergence and robustness.

10. D. E. Goldberg 1989 Genetic Algorithms in Search, Optimization and Machine Learning. Cambridge, MA: Addison-Wesley.

11. T. Bäck, D. B. Fogel and Z. Michalewicz (editors) 1997. Handbook of Evolutionary Computation. Bristol: IOP Publishing Ltd, Oxford: Oxford University Press.

12. K. Shankar and A. J. Keane 1995 Journal of Sound and Vibration 185, 867-890. Energy flow predictions in a structure of rigidly joined beams using receptance theory.

13. T. BÄCK 1996 Evolutionary Algorithms in Theory and Practice. Oxford: Oxford University Press.

14. J. N. Siddall 1982 Optimal Engineering Design: Principles and Applications. New York: Marcel Dekker, Inc.

15. W. H. Press, S. A. Teukolsky and W. T. Vetterling 1992 Numerical Recipes in FORTRAN 77: The Art of Scientific Computing. Cambridge: Cambridge University Press. 